\title{
Computing argumentation for decision making in legal disputes
}

\author{
Maxime Morge \\ Dipartimento di Informatica, Università di Pisa \\ via F. Buonarroti, 2 I-56127 Pisa, Italy \\ morge@di.unipi.it, \\ http://maxime.morge.org
}

\begin{abstract}
In this paper, we present a decision support system for lawyer. This system is built upon an argumentation framework for decision making. A logic language is used as a concrete data structure for holding the statements like knowledge, goals, and decisions. Different priorities are attached to these items corresponding to the uncertainty of the knowledge about the circumstances, the lawyer's preferences, and the expected utilities of sentences. These concrete data structures consist of information providing the backbone of arguments. Due to the abductive nature of practical reasoning, arguments are built by reasoning backwards, and possibly by making suppositions over missing information. Moreover, arguments are defined as tree-like structures. In this way, our computer system, implemented in Prolog, suggests some actions and provides an interactive and intelligible explanation of this solution.
\end{abstract}

\section{Introduction}

Since legal disputes are resolved by confronting and evaluating the justifications of parties' positions, argumentation is central to law. This is the reason why many works in the area of Artificial Intelligence \& Law focus on the computational model of argumentation. In particular, nonmonotonic logic techniques have been used to model the vagueness, indeterminacy and adversarial nature of the law with hierarchies of possibly conflicting rules (see [1] for a survey). However, even if modern techniques are used, this logical approach is still limited to the epistemic reasoning and do not encompass practical reasoning. The point is that a legal dispute in criminal cases is not only limited to draw conclusions (e.g. the guilt or the innocence of an accused) but must determine a sentence, i.e. take a decision.

In this paper, we present a decision support system for lawyer. This system is built upon an Argumentation Framework (AF) for decision making. A logic language is used as a concrete data structure for holding the statements like knowledge, goals, and decisions. Different priorities are attached to these items corresponding to the uncertainty of the knowledge about the circumstances, the lawyer's preferences, and the expected utilities of sentences. These concrete data structures consist of information providing the backbone of arguments. Due 
to the abductive nature of practical reasoning, arguments are built by reasoning backwards, and possibly by making suppositions over missing information. Moreover, arguments are defined as tree-like structures. In this way, our computer system, implemented in Prolog, suggests some actions and provides an interactive and intelligible explanation of this solution.

Section 2 introduces the walk-through example. In order to present our AF, we will browse the following fundamental notions. Firstly, we define the object language (cf Section 3) and the associated priorities (cf Section 4). Secondly, we will focus on the internal structure of arguments (cf Section 5). We present in Section 6 the interactions between them. These relations allow us to give a declarative model-theoretic semantics to our AF (cf Section 7) and we adopt a dialectical proof procedure to implement it (cf Section 8). Section 9 discusses some related works. Section 10 draws some conclusions and directions for future work.

\section{Walk-through example}

Inspired by [2], we consider here criminal sentencing. Such a decision making problem requires a proper understanding of all relevant aspects. The goals for the sentences such as the punishment, the deterrence, the rehabilitation, and so on, as well as the knowledge about the surrounding circumstances, such as the influence of alcohol or drugs is also of vital importance. The judge is responsible for sentencing, based on the explicit goals and on her knowledge.

We assume that the user provides, via the GUI, influence diagrams [3]. These are simple graphical representations of multi-attribute decision problems. Here, they are used by the judge to display the structure of the decision problem related to the criminal case. In addition, the GUI allows the justifications to communicate specific details, in particular facts and preferences.

In legal disputes about criminal cases, the main goal, that consists in an appropriate sentence (denoted $\mathrm{jdg}$ ), is addressed by a set of decisions, i.e. a choice amongst some sentences. The accused can (or cannot) be put in prison (Prison(yes), Prison(no)), The accused can (or cannot) do a community service (Service(yes) or Service(no)). The accused can (or cannot) be fine without payement (Fine(yes) or Fine(no)). The main goal is split into independent subgoals. The judgement must punish the offender (pu), rehabilitating the offender (re), protecting the society from crime (pt), and deterring the general public (de). The knowledge about the crime is expressed with predicates such as: guilty (the accused is found guilty), alcohol/drug (the crime is influenced by alcohol/drugs), driving (the crime is driving related) or mobile (the criminal was using a mobile phone $\left.{ }^{1}\right)$.

Figure 1 provides a simple graphical representation of the decision problem called influence diagram. The elements of the decision problem, i.e. values (represented by rectangles with rounded corners), decisions (represented by rectangles)

\footnotetext{
${ }^{1}$ We consider here the particular french jurisdiction where the usage of mobile is
} forbidden during driving. 
and knowledge (represented by ovals), are connected by arcs where predecessors affect successors. We consider here a multiattribute decision problem captured by a hierarchy of values where the abstract value (represented by rectangles with rounded corner and double line) aggregates the values in the lower level. When the structure of the decision is built, the alternatives must be identified, the preferences must be expressed and the knowledge gathered.

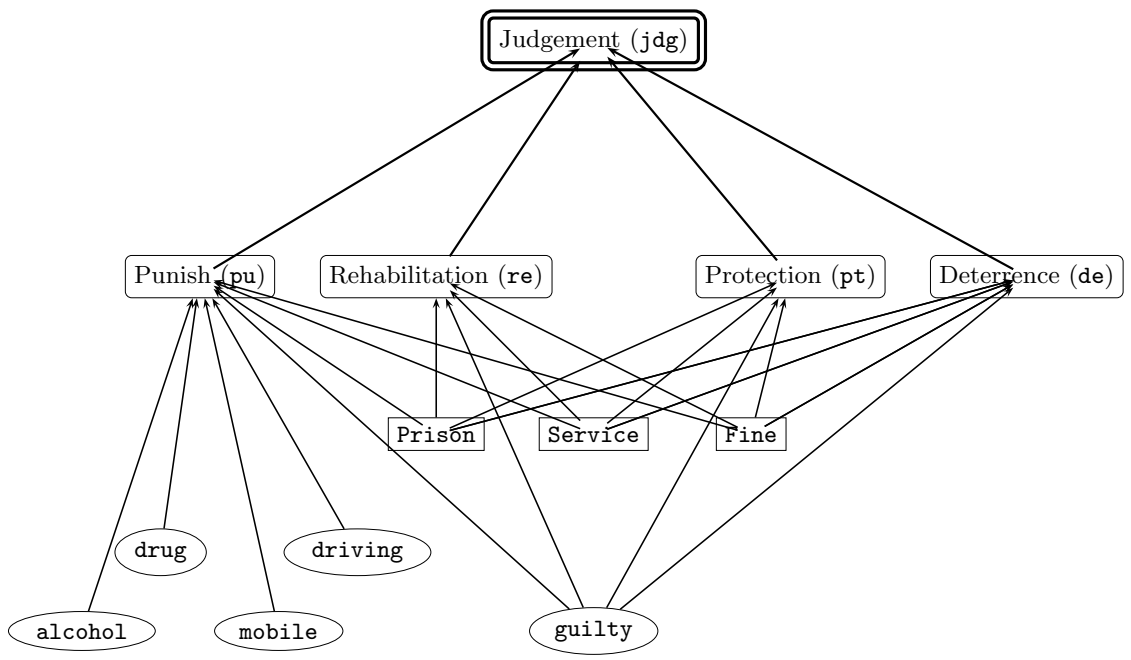

Fig. 1. Influence diagram for criminal sentencing

The judge also provides, through the GUI, the facts and her preferences. For example, the judge knows that the crime was influenced by alcohol and she does not know if the crime was made during the usage of a mobile. Due to conflicting sources of information, the judge has conflicting information about the influence of drugs and the fact that the crime is driving related. These sources of information are more or less reliable. The judge can (or cannot) have preferences over them.

In order to represent the structure of the decision and to express preferences and constraints, an object language is required.

\section{The object language}

Since we want to provide a computational model of argumentation for decision making and we want to instantiate it for our example, we need to specify a particular logic. 
The object language expresses rules and facts in logic-programming style. In order to address a decision making problem, we distinguish:

- a set of abstract goals, i.e. some propositional symbols which represent the abstract features that the decisions must exhibit (in the example $\mathrm{jdg}$ is the only abstract goal);

- a set of concrete goals, i.e. some propositional symbols which represent the concrete features that the decisions must exhibit (in the example pu, re, pt and de);

- a set of decisions, i.e. some predicate symbols which represent the actions which must be performed or not (in the example Prison, Service, and Fine);

- a set of alternatives, i.e. some constants symbols which represent the mutually exclusive solutions for each decision (in the example yes, or no);

- a set of beliefs, i.e. some propositional symbols which represent epistemic statements (in the example guilty, alcohol, ...). In the language, we explicitly distinguish assumable beliefs (respectively non-assumable) beliefs, which can (respectively cannot) be taken for granted. Since we cannot make the supposition that the accused is guilty, guilty is non-assumable.

Since we want to consider conflicts in this object language, we need some forms of negation. For this purpose, we consider strong negation, also called explicit or classical negation, and weak negation, also called negation as failure. A strong literal is an atomic first-order formula, possible preceded by strong negation $\neg$. A weak literal is a literal of the form $\sim L$, where $L$ is a strong literal. $\neg L$ says "L is definitely not the case", while $\sim L$ says "There is no evidence that $\mathrm{L}$ is the case". In order to express in a compact way the mutual exclusion between statements, such as the different alternatives for a decision, we define the incompatibility relation (denoted by $\mathcal{I}$ ) as a binary relation over atomic formulas which is asymmetric. Whatever the atom $L$ is a belief or a goal, we have $L \mathcal{I} \neg L$ and $\neg L \mathcal{I} L$, while we have $L \mathcal{I} \sim L$ but we do not have $\sim L \mathcal{I} L$. Obviously, $D_{1}\left(a_{1}\right) \mathcal{I} D_{1}\left(a_{2}\right)$ and $D_{1}\left(a_{2}\right) \mathcal{I} D_{1}\left(a_{1}\right), D_{1}$ being a decision predicate, $a_{1}$ and $a_{2}$ being different ${ }^{2}$ alternatives for $D$. Moreover, some sentences can be incompatible. For instance, Prison(yes) $\mathcal{I}$ Fine(yes) and Fine(yes) $\mathcal{I}$ Prison(yes) but neither Prison(no) $\mathcal{I}$ Fine(no) and nor Fine(no) $\mathcal{I}$ Prison(no). Similarly, we say that two sets of sentences $\Phi_{1}$ and $\Phi_{2}$ are incompatible $\left(\Phi_{1} \mathcal{I} \Phi_{2}\right)$ iff there is a sentence $\phi_{1}$ in $\Phi_{1}$ and a sentence $\phi_{2}$ in $\Phi_{2}$ such as $\phi_{1} \mathcal{I} \phi_{2}$. A theory gathers the statements about the decision making problem.

Definition 1 (Theory). A theory $\mathcal{T}$ is an extended logic program, i.e a finite set of rules such as $R: L_{0} \leftarrow L_{1}, \ldots, L_{j}, \sim L_{j+1}, \ldots, \sim L_{n}$ with $n \geq 0$, each $L_{i}$ being a strong literal. The literal $L_{0}$, called the head of the rule, is denoted head $(R)$. The finite set $\left\{L_{1}, \ldots, \sim L_{n}\right\}$, called the body of the rule, is denoted $\operatorname{bod} y(R)$. The body of a rule can be empty. In this case, the rule, called a fact,

\footnotetext{
${ }^{2}$ Notice that in general a decision can be addressed by more than two alternatives.
} 
is an unconditional statement. $R$, called the name of the rule, is an atom in the language $\mathcal{L}$. All rules are ground.

Considering a decision making problem, we distinguish:

- goal rules of the form $R: G_{0} \leftarrow G_{1}, \ldots, G_{n}$ with $n>0$. Each $G_{i}$ is a goal literal (or its negation). According to this rule, the goal is promoted (or demoted) by the combination of goal literals in the body;

- epistemic rules of the form $R: B_{0} \leftarrow B_{1}, \ldots, B_{n}$ with $n \geq 0$. Each $B_{i}$ is a belief literal. According to this rule, the belief $B_{0}$ is true if the conditions $B_{1}, \ldots, B_{n}$ are satisfied;

- decision rules of the form $R: G \leftarrow D(a), B_{1}, \ldots, B_{n}$ with $n \geq 0$. The head of the rule is a concrete goal (or its strong negation). The body includes a decision literal $(D(a))$ and a possible empty set of belief literals. According to this rule, the goal is promoted (or demoted) by the decision $D(a)$, provided that conditions $B_{1}, \ldots, B_{n}$ are satisfied.

Considering statements in the theory is not sufficient to take a decision.

\section{Priority}

In order to evaluate the previous statements, all relevant pieces of information should be taken into account, such as the likelihood of beliefs, the preferences between goals, or the expected utilities of the decisions.

In Mathematics, order relations are binary relations on a set. Since these relations classify the elements from the 'best' to the 'worst', with or without ex aquo, they are qualitative. For this purpose, we can consider either a preorder, i.e. a reflexive and transitive relation considering possible ex aquo, or an order, i.e. an antisymmetric preorder relation. The preorder (respectively the order) is total iff all elements are comparable. In this way, we consider that the priority $\mathcal{P}$ is a (partial or total) preorder on the rules in $\mathcal{T} . R_{1} \mathcal{P} R_{2}$ can be read " $R_{1}$ has priority over $R_{2}$ ". $R_{1} \ngtr R_{2}$ can be read " $R_{1}$ has no priority over $R_{2}$ ", either because $R_{1}$ and $R_{2}$ are ex aquo (denoted $R_{1} \sim R_{2}$ ), i.e. $R_{1} \mathcal{P} R_{2}$ and $R_{2} \mathcal{P} R_{1}$, or because $R_{1}$ and $R_{2}$ are not comparable, i.e. $\neg\left(R_{1} \mathcal{P} R_{2}\right)$ and $\neg\left(R_{2} \mathcal{P} R_{1}\right)$.

In this work, we consider that all rules are potentially defeasible and that the priorities are extra-logical and domain-specific features. The priority over concurrent rules depends of the nature of rules. Rules are concurrent if their heads are identical or incompatible. We define three priority relations:

- the priority over goal rules comes from the preferences overs goals. The priority of such rules corresponds to the relative importance of the combination of (sub)goals in the body as far as reaching the goal in the head is concerned;

- the priority over epistemic rules comes from the uncertainty of knowledge. The prior the rule is, the more likely the rule holds;

- the priority over decision rules comes from the expected utility of decisions. The priority of such rules corresponds to the expectation of the conditional decision in promoting the goal literal. 
In order to illustrate the notions introduced previously, let us consider the example. The goal theory, the epistemic theory, and the decision theory are represented in Table 1 . A rule above another one has priority over it. To simplify the graphical representation of the theories, they are stratified in non-overlapping subsets, i.e. different levels. The ex aquo rules are grouped in the same level. Non-comparable rules are arbitrarily assigned to a level.

$\left\{\begin{array}{l}r_{01}: \mathrm{jdg} \leftarrow \mathrm{pu}, \mathrm{re}, \mathrm{pt}, \neg \mathrm{de} \\ \hline r_{02}: \mathrm{jdg} \leftarrow \mathrm{pu}, \mathrm{re}, \neg \mathrm{de} \\ \hline r_{03}: \mathrm{jdg} \leftarrow \mathrm{pu}, \neg \mathrm{re}, \mathrm{pt}, \mathrm{de} \\ r_{04}: \mathrm{jdg} \leftarrow \mathrm{pu}, \mathrm{re}, \mathrm{de}\end{array}\right.$

$$
\begin{aligned}
& f_{0}: \text { driving } \leftarrow \\
& f_{1}: \text { guilty } \leftarrow \\
& f_{2}: \text { drug } \leftarrow \\
& f_{2}^{\prime}: \neg \text { drug } \leftarrow \\
& f_{3}: \text { alcohol } \leftarrow \\
& \hline f_{0}^{\prime}: \neg \text { driving } \leftarrow
\end{aligned}
$$

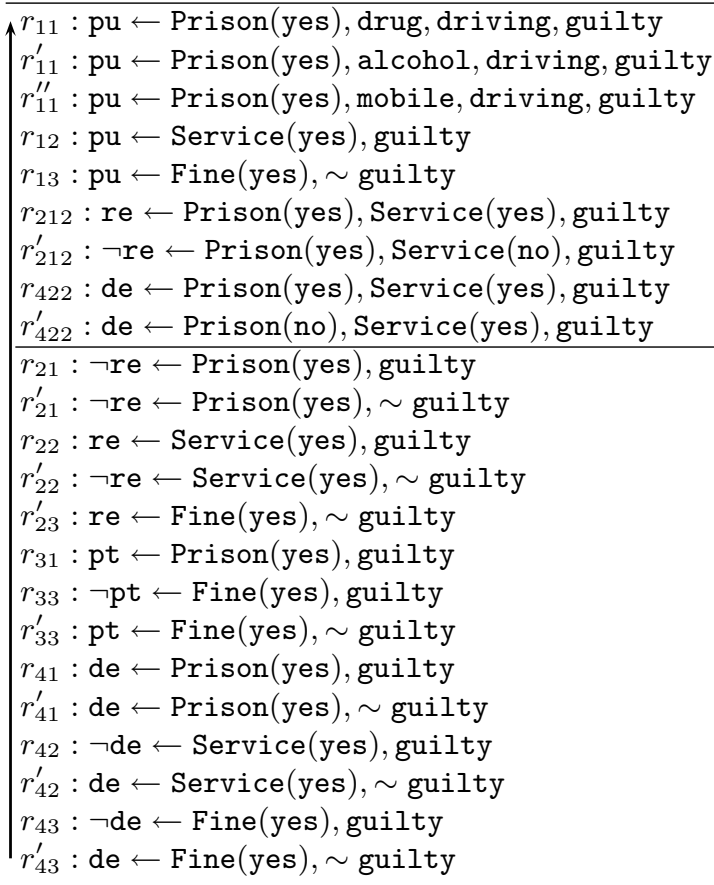

Table 1. The goal theory (at upper left), the epistemic theory (at lower left), and the decision theory (at right).

According to the decision theory, the community service is relevant to punish a criminal $\left(r_{12}\right)$, being fine is relevant for the (non)punishment of an innocent $\left(r_{13}\right)$, and the prison is relevant to punish a driving related crime influenced by drugs $\left(r_{11}\right)$ or alcohol $\left(r_{11}^{\prime}\right)$, or made during the usage of a mobile $\left(r_{11}^{\prime \prime}\right)$. Actually, the utilities of these alternatives with respect to pu depends on the surrounding circumstances. "Do a community service" is stronger in promoting re than "go to prison" in demoting re $\left(r_{212} \mathcal{P} r_{21}\right)$. Similarly, "go to prison" is stronger in promoting de than "do a community service" in demoting deterrence $\left(r_{422} \mathcal{P} r_{42}\right)$. Our formalism allows to capture the mutual influence of decisions over the independent goals. 
According to the goal theory, achieving the goals pu, re, and pt and avoiding de is required to reach $\mathrm{jdg}\left(\mathrm{cf} r_{01}\right)$. However, these constraints can be relaxed. We make pu an essential goal by requiring it also in $r_{02}$, in $r_{03}$, and in $r_{04}$. The achievement of pt can be relaxed $\left(r_{01} \mathcal{P} r_{02}\right)$. Moreover, the achievement of re is more important than de and pt put together $\left(r_{01} \mathcal{P} r_{03}\right.$ and $\left.r_{02} \mathcal{P} r_{03}\right)$ and promoting re while demoting de is preferable to promoting de $\left(r_{02} \mathcal{P} r_{04}\right)$. Our formalism allows to capture complex and incomplete information about the preferences amongst goals.

According to the epistemic theory, the judge finds the accused guilty $\left(f_{1}\right)$, knows that the crime was influenced by alcohol $\left(f_{3}\right)$, and does not know if the crime was made during the usage of a mobile. Due to conflicting sources of information, the judge has conflicting information about the influence of drugs $\left(f_{2}\right.$ and $\left.f_{2}^{\prime}\right)$ and the fact that the crime is driving related $\left(f_{0}\right.$ and $\left.f_{0}^{\prime}\right)$. The sources of information can be more or less reliable. For instance, we have $f_{0} \mathcal{P} f_{0}^{\prime}$ but there is no strict priority between $f_{2}$ and $f_{2}^{\prime}$. Our formalism allows to capture complex (and incomplete) information about the likelihood of the surrounding circumstances. We will build now arguments upon these (incomplete) statements in order to compare the alternatives.

\section{Arguments}

Due to the abductive nature of the practical reasoning, we define and construct arguments by reasoning backwards, and possibly by making suppositions over missing information. Since we adopt a tree-like structure of arguments, our framework not only suggests some actions but also provides an intelligible explanation of them.

The simplest way to define an argument is by a pair 〈premises, conclusion > as in [4]. This definition leaves implicit that the underlying logic validates a proof of the conclusion from the premises. When the argumentation framework is built upon an extended logic program, an argument is often defined as a sequence of rules [5]. These definitions ignore the recursive nature of arguments: arguments are composed of subarguments, subarguments for these subarguments, and so on. For this purpose, we adopt the tree-like structure for arguments proposed in $[6]$ and we extend it with suppositions on the missing information.

Definition 2 (Argument). An argument is composed by a conclusion, a top rule, some premises, some suppositions, and some sentences. These elements are abbreviated by the corresponding prefixes. An argument $A$ is:

1. a hypothetical argument built upon an unconditional ground statement. If $L$ is a assumable belief, then the argument built upon this assumable belief is defined as follows ${ }^{3} \operatorname{conc}(A)=L, \operatorname{top}(A)=\theta, \operatorname{premise}(A)=\emptyset, \operatorname{supp}(A)=$ $\{L\}, \operatorname{sent}(A)=\{L\}$.

or

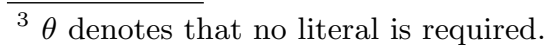


2. a built argument built upon a rule such that all the literals in the body are the conclusion of subarguments.

1) If $f$ is a fact in $\mathcal{T}$ (i.e. body $(f)=\emptyset$ ), then the trivial argument $A$ built upon this fact is defined as follows: $\operatorname{conc}(A)=$ head $(f), \operatorname{top}(A)=f$, $\operatorname{premise}(A)=\emptyset, \operatorname{supp}(A)=\emptyset, \operatorname{sent}(A)=\{$ head $(f)\}$.

2) If $r$ is a rule in $\mathcal{T}$, we define the tree argument $A$ built upon this rule as follows. Let body $(r)=\left\{L_{1}, \ldots, L_{j}, \sim L_{j+1}, \sim L_{n}\right\}$ and $\operatorname{sbarg}(A)=$ $\left\{A_{1}, \ldots, A_{n}\right\}$ be the collection of arguments such that, for each strong literal $L_{i} \in \operatorname{body}(r), \operatorname{conc}\left(A_{i}\right)=L_{i}$ with $i \leq j$ or $\operatorname{conc}\left(A_{i}\right)=\sim L_{i}$ with $i>j$ (each $A_{i}$ is called a subargument of $A$ ). Then: $\operatorname{conc}(A)=$ head $(r), \operatorname{top}(A)=$ $r, \operatorname{premise}(A)=\operatorname{body}(r), \operatorname{supp}(A)=\cup_{A^{\prime} \in \operatorname{sbarg}(A)} \operatorname{supp}\left(A^{\prime}\right), \operatorname{sent}(A)=$ $\cup_{A^{\prime} \in \operatorname{sbarg}(A)} \operatorname{sent}\left(A^{\prime}\right) \cup \operatorname{body}(r) \cup\{$ head $(r)\}$.

As in [6], we consider composite arguments (3) and atomic arguments (2) where the top rule is a fact. Contrary to the other definitions of arguments (pair of premises - conclusion, sequence of rules), our definition considers that the different premises can be challenged and can be supported by subarguments. In this way, arguments are intelligible explanations. Moreover, we distinguish hypothetical arguments (1) and built arguments (2/3). While built arguments are built upon a top rule which is a rule or a fact of the theory, hypothetical arguments are built upon missing information. In this way, our framework allows to reason further by making suppositions related to the unknow beliefs and over possible decisions under which arguments can be built. Due to the abductive nature of practical reasoning, we define and construct arguments by reasoning backwards. Therefore, arguments do not include irrelevant information such as sentences not used to derive the conclusion.

Let us consider the previous example. Some of the arguments concluding pu are depicted in Figure 2. According to the argument $B$, the accused will be punish if he is guilty and we suppose that he does a community service. According to the argument $A_{1}$ (respectively $A_{2}$ ), the accused will be punish if he is guilty of a driving-related crime, if we suppose he goes in Prison, and if he is influence by drugs (respectively we suppose he was using a mobile phone). An argument can be represented as tree where the root is the conclusion (represented by a triangle) directly connected to the premises (represented by losanges) if they exist, and where the leefs are either some suppositions (represented by circles) or emptyset. Each plain arrow corresponds to a rule (or a fact) where the head node corresponds to the head of the rule and the tall nodes are in the body of the rule. The tree argument $A^{1}$ is composed of four subarguments: one hypothetical argument and three trivial arguments. The tree argument $A^{2}$ is composed of four subarguments: two hypothetical arguments and two trivial arguments. Neither trivial arguments nor hypothetical arguments contain subarguments. Due to their structures and their natures, arguments interact with one another. 

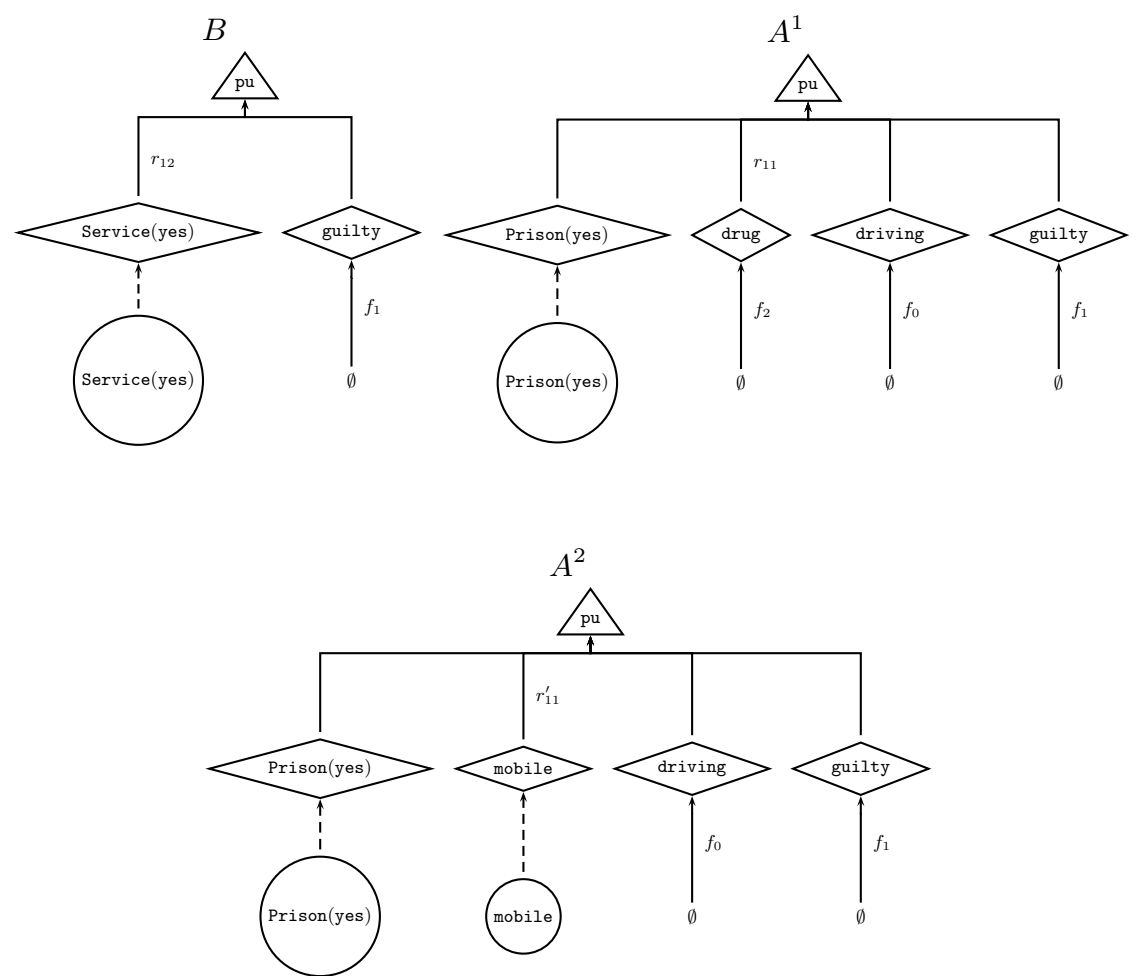

Fig. 2. Some arguments concluding pu 


\section{Interactions between arguments}

The interactions between arguments may come from the incompatibility of their sentences, from their nature (hypothetical or built) and from the priority over rules. We examine in turn these different sources of interaction.

Since their sentences are conflicting, arguments interact with one another. For this purpose, we define the attack relation.

Definition 3 (Attack relation). Let $A$ and $B$ be two arguments. $A$ attacks $B$ (denoted by attacks $(A, B))$ iff $\operatorname{sent}(A) \mathcal{I} \operatorname{sent}(B)$.

This relation encompasses both the direct (often called rebuttal) attack due to the incompatibility of the conclusions, and the indirect (often called undermining) attack, i.e. directed to a "subconclusion". According to this definition, if an argument attacks a subargument, the whole argument is attacked. The attack relation is useful to build arguments which are homogeneous explanations.

Due to the nature of argument, arguments are more or less hypothetical. This is the reason why we define the size of their suppositions.

Definition 4 (Supposition size). Let $A$ be an arguments. The size of suppositions for $A$, denoted suppsize $(A)$, is defined such that:

1. if $A$ is a hypothetical argument, then suppsize $(A)=1$;

2. if $A$ is a trivial argument, then suppsize $(A)=0$;

3. if $A$ is a tree argument and $\operatorname{sbarg}(A)=\left\{A_{1}, \ldots, A_{n}\right\}$ is the collection of subarguments of $A$, then suppsize $(A)=\Sigma_{A^{\prime} \in \operatorname{sbarg}(A)} \operatorname{suppsize}\left(A^{\prime}\right)$.

The size of suppositions for an argument does not only count the number of hypothetical subarguments which compose the argument but also counts the number of hypothetical subarguments of these subarguments, and so on.

Since arguments have different natures (hypothetical or built) and the top rules of built arguments are more or less strong, they interact with one another. For this purpose, we define the strength relation.

Definition 5 (Strength relation). Let $A_{1}$ be a hypothetical argument, and $A_{2}, A_{3}$ be two built arguments.

1. $A_{2}$ is stronger than $A_{1}$ (denoted $A_{2} \quad \mathcal{P} \mathcal{A} A_{1}$ );

2. If $\left(\operatorname{top}\left(A_{2}\right) \mathcal{P}\right.$ top $\left.\left(A_{3}\right)\right) \wedge \neg\left(\operatorname{top}\left(A_{3}\right) \mathcal{P}\right.$ top $\left.\left(A_{2}\right)\right)$, then $A_{2} \quad \mathcal{P} \mathcal{A} A_{3}$;

3. If $\left(\operatorname{top}\left(A_{2}\right) \mathcal{R}\right.$ top $\left.\left(\mathrm{A}_{3}\right)\right) \wedge\left(\operatorname{suppsize}\left(\mathrm{A}_{2}\right)<\operatorname{suppsize}\left(\mathrm{A}_{3}\right)\right)$, then $A_{2} \mathcal{P} \mathcal{A} A_{3}$;

Since $\mathcal{P}$ is a preorder on $\mathcal{T}, \quad \mathcal{P} \mathcal{A}$ is a preorder on $\mathcal{A}(\mathcal{T})$. Built arguments are preferred to hypothetical arguments. An argument is stronger than another argument if the top rule of the first argument has a proper higher priority that the top rule of the second argument or if the top rule of the first argument does not have a proper higher priority but the number of suppositions made in the first argument is properly smaller than the number of suppositions made in the second argument. The strength relation is useful to choose (when it is possible) 
between homogeneous concurrent explanations, i.e. non conflicting arguments with the same conclusions.

The two previous relations can be combined to choose (if possible) between non-homogeneous concurrent explanations, i.e. conflicting arguments with the same conclusions.

Definition 6 (Defeats). Let $A$ and $B$ be two arguments. $A$ defeats $B$ (written defeats $(A, B))$ iff:

1. $\operatorname{attacks}(A, B)$;

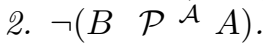

Similarly, we say that a set $S$ of arguments defeats an argument $A$ if $A$ is defeated by one argument in $S$.

Let us consider our previous example. The arguments in favor of prison $\left(A^{1}\right.$ and $A^{2}$ ) and the argument in favor of community service $(B)$ attack each other. Since the top rule of $A^{1}$ (i.e. $R_{11}$ ), the top rule of $A^{2}$ (i.e. $R_{11}^{\prime \prime}$ ), and the top rule of $B$ (i.e. $R_{12}$ ) are not stronger than each other, $A^{1} / A^{2}$ defeat $B$ and $B$ defeats $A^{1} / A^{2}$. If we only consider these three arguments, the judge cannot decide what the best alternatives are, and the best arguments to explain the choices. However, $A^{1}$, which is composed of one hypothetical argument and three trivial arguments, is "better" than $A^{2}$, which is composed of two hypothetical arguments and two trivial arguments. Determining whether a suggestion and an explanation are ultimately suggested requires a complete analysis of all arguments and subarguments. In this section, we have defined the interactions between arguments in order to give them a status.

\section{Semantics}

We can consider our AF abstracting away from the logical structures of arguments. This abstract AF consists of a set of arguments associated with a binary defeat relation.

Given an AF, [7] and [8] define the following notions of "acceptable" sets of arguments:

Definition 7 (Semantics). An $A F$ is a pair $\langle\mathcal{A}$, defeats $\rangle$ where $\mathcal{A}$ is a set of arguments and defeats $\subseteq \mathcal{A} \times \mathcal{A}$ is the defeat relationship ${ }^{4}$ for $A F$. For $A \in \mathcal{A}$ an argument and $S \subseteq \mathcal{A}$ a set of arguments, we say that:

- $A$ is acceptable with respect to $S$ (denoted $A \in \mathcal{S}_{\mathcal{A}}^{S}$ ) iff $\forall B \in \mathcal{A}$, defeats $(B, A)$ $\exists C \in S$ such that defeats $(C, B)$;

- $S$ is conflict-free iff $\forall A, B \in S \neg$ defeats $(A, B)$;

- $S$ is admissible iff $S$ is conflict-free and $\forall A \in S, A \in \mathcal{S}_{\mathcal{A}}^{S}$;

- $S$ is preferred iff $S$ is maximally admissible;

\footnotetext{
${ }^{4}$ Actually,the defeat relation is called attack in [7] and in [8].
} 
- $S$ is complete iff $S$ is admissible and $S$ contains all arguments $A$ such that $S$ defeats all defeaters against $A$;

- $S$ is grounded iff $S$ is minimally complete;

$-S$ is ideal iff $S$ is admissible and it is contained in every preferred sets.

The semantics of an admissible (or preferred) set of arguments is credulous, in that it sanctions a set of arguments as acceptable if it can successfully dispute every arguments against it, without disputing itself. However, there might be several conflicting admissible sets. Various sceptical semantics have been proposed for AF, notably the grounded semantics, the ideal semantics, and the sceptically preferred semantics, whereby an argument is accepted if it is a member of all maximally admissible sets of arguments.

Since some ultimate choices amongst various admissible sets of alternatives are not always possible, we consider in this paper only the credulous semantics. Let us focus on the goal pu in the previous example. Since $\left\{A^{1}\right\},\{B\}$ are admissible and $\left\{A^{2}\right\}$ is not admissible, different alternatives and explanations for different decisions can be suggested to reach pu. If we consider now the whole problem, the argument depicted in Figure 3 is the only one reaching jdg which is admissible.

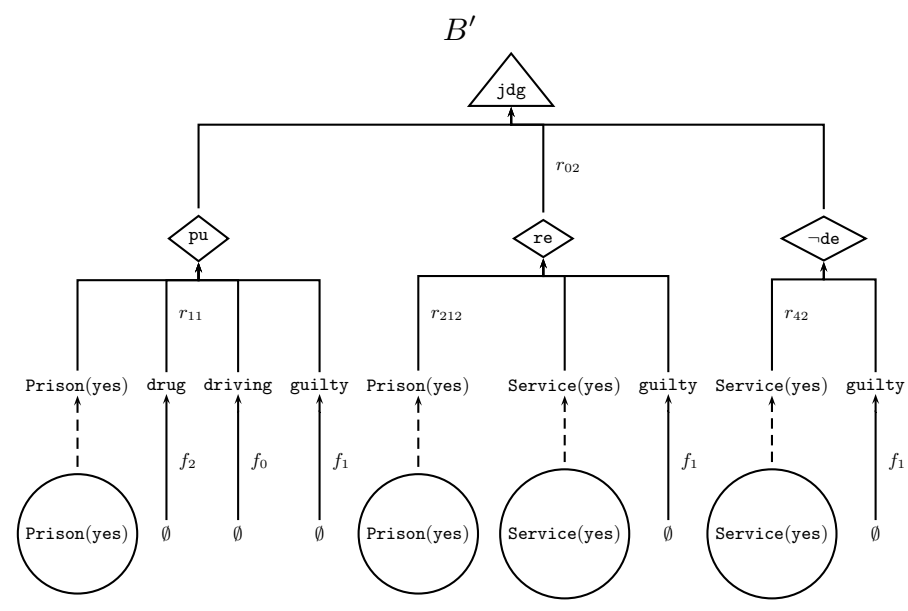

Fig. 3. Some arguments concluding jdg

In our example, there is only one admissible argument deriving the main goal. However, in the general case, a decision $D_{1}\left(a_{1}\right)$ is suggested iff $D_{1}\left(a_{1}\right)$ is a supposition of one argument in an admissible set deriving the main goal. Therefore, our AF involves some ultimate choices of the lawyer between various admissible sets of alternatives. In this section, we have given a status to the arguments. 


\section{Procedure}

A dialectical proof procedure is required to compute the model-theoretic semantics of our argumentation framework. The procedures proposed in $[8,9]$ compute the credulous semantics. Since our practical application requires to specify the internal structure of arguments, we adopt the procedure proposed in [8].

In order to compute admissible arguments in our AF, we have translated our $\mathrm{AF}$ in an Assumption-based AF (ABF for short). $\mathrm{CaSAPI}^{5}$ [10] computes the admissible semantics in the ABF by implementing the procedure originally proposed in [11]. Suppose we wish to investigate whether an argument is preferred, i.e. it belongs to a preferred set. We know that it suffices to check that this argument is in an admissible set, since, by definition, a preferred set is a maximal admissible set and obviously all admissible sets are contained in a maximal admissible set. If the procedure succeeds, we know that the argument is contained in a preferred set. Moreover, we have developed a CaSAPI meta-interpreter to relax the goals achievements in the priority order and to make suppositions in order to compute the admissible semantics in our concrete $\mathrm{AF}^{6}$. We can easily extend it to compute the competing semantics which have been proposed in [8]. The implementation of our framework, called MARGO (Multiattribute ARGumentation framework for Opinion explanation), is written in Prolog and available in GPL (GNU General Public License) at http://margo. sourceforge.net/. In this section, we have shown how to compute admissible arguments in our AF.

\section{Related works}

Argumentation has been put forward as a promising approach to support decision making [12]. While influence diagrams and belief networks [13] require that all the factors relevant for a decision are identified a priori, arguments are defeasible or reinstantiated in the light of new information not previously available.

Contrary to the theoretical reasoning, practical reasoning is not only about whether some beliefs are true, but also about whether some actions should or should not be performed. The practical reasoning [14] follows three main steps: i) deliberation, i.e. the generation of goals; ii) means-end reasoning, i.e. the generation of plans; iii) decision-making, i.e. the selection of plans that will be performed to reach the selected goals. For instance, [15] proposes an AF focusing on the deliberation (closed to the principle of [16] where argumentation is implicit) and $[17,18]$ have provided formal models for deliberation and means-end reasoning. While some frameworks are based upon defeasible logic programming (e.g. $[19,20])$, most of them instantiate the abstract argumentation framework of Dung [7]. Since the latter abstracts away from the internal structure of arguments in order to focus on the manner in which arguments interact, [21] instantiates

\footnotetext{
${ }^{5}$ http://www.doc.ic.ac.uk/ ${ }^{\sim}$ dg00/casapi.html

${ }^{6}$ For brevity, we do not describe this mechanism in the paper.
} 
an argument scheme in the context of practical reasoning in order to capture the interaction in terms of internal structure.

In this work, we have proposed an AF for decision-making. In this perspective, [22] proposes a critical survey of some computational models of argumentation over actions. For this purpose, $[23,24]$ have considered several principles according to the different types of arguments which are considered are aggregated. However, contrary to our approach, the potential interaction amongst arguments, as studied in the seminal work of Dung [7] is not considered. In this paper we have extended the legal example borrowed from [2] and we have adopted like [2] an abductive approach to the practical reasoning which is directly modelled within in our framework.

Finally, to the best of our knowledge, few implementation of argumentation over actions exist. CaSAPI and DeLP 7 are restricted to the theoretical reasoning. PARMENIDES ${ }^{8}$ is a software to structure the debate over actions by adopting a particular argumentation scheme. GORGIAS ${ }^{9}$ implements an argumentation based framework to support the decision making of an agent within a modular architecture. Like the latter, MARGO incorporate abduction on missing information. Moreover, we can easily extend it to compute the competing semantics which have been proposed in [8] since we have instantiated the abstract argumentation framework of Dung.

\section{Conclusions}

In this paper we have presented a concrete and implemented AF for practical reasoning in legal disputes which suggests different alternative courses of actions and provides an interactive and intelligible explanation of the choices. A logic language is used as a concrete data structure for holding the statements like knowledge, goals, and decisions. Different priorities are attached to these items corresponding to the uncertainty of the knowledge about the circumstances, the lawyer's preferences between goals, and the expected utilities of sentences. These concrete data structures consist of information providing the backbone of arguments. Due to the abductive nature of practical reasoning, arguments are built by reasoning backwards, and possibly by making suppositions over missing information. To be intelligible, arguments are defined as tree-like structures. The interactions between arguments may come from the incompatibility of their sentences, from their nature (hypothetical or built) and from the priority over rules. Since an ultimate choice amongst various admissible sets of alternatives is not always possible, we have adopted a credulous semantics. In order to compute it, we have implemented our AF in Prolog.

In future works, we want to incorporate decision-theoretic techniques within the model. Standard decision theory weighs the cost and benefits of possible outcomes with their probabilities to produce a preference on the expected utilities

\footnotetext{
${ }^{7}$ http://lidia.cs.uns.edu.ar/DeLP

${ }^{8}$ http://cgi.csc.liv.ac.uk/ katie/Parmenides.html

9 http://www.cs.ucy.ac.cy/ nkd/gorgias/
} 
of the alternatives. However in many practical applications, it is not natural to give a quantitative representation of many objectives, or it could not deal with the cases of decision makers that only have partial information. Further standard decision theory provides little support in giving intelligible explanation of the choices. For this purpose, it would be best to have a hybrid approach combining both quantitative and qualitative decision theory. Argumentation provides a natural framework for these hybrid systems by providing a link between qualitative objectives and its quantitative representation. In addition, sentencing is usually governed by some explicit statutory or regulatory rules. We want to take into account them in our framework.

\section{Acknowledgements}

We would like to thank the anonymous reviewers for their detailed comments on this paper. The author would like to thank Paolo Mancarella, Phan Minh Dung and Francesca Toni for their comments on a previous version of this paper. This work is supported by the Sixth Framework IST programme of the EC, under the 035200 ARGUGRID project.

\section{References}

1. Prakken, H., Sartor, G.: The role of logic in computational models of legal argument: a critical survey. Number 2408 in Lecture Notes in Computer Science. In: Computational Logic: Logic Programming and Beyond. Essays In Honour of Robert A. Kowalski, Part II. Antonis Kakas and Farida Sadri edn. Springer, Berlin (2002) 343-380

2. Bench-Capon, T., Prakken, H.: Justifying actions by accruing arguments. In: Proc. of the 1st International Conference on Computational Models of Argument, IOS Press (2006) 247-258

3. Clemen, R.T.: Making Hard Decisions. Duxbury. Press (1996)

4. Amgoud, L., Cayrol, C.: A reasoning model based on the production of acceptable arguments. Annals of Maths and AI 34(1-3) (2002) 197-215

5. Ralf Schweimeier, M.S.: Notions of attack and justified arguments for extended logic programs. In van Harmelen, F., ed.: Proc. of the 15th European Conference on Artificial Intelligence (ECAI), Amsterdam, IOS Press (2002) 536-540

6. Vreeswijk, G.: Abstract argumentation systems. Artificial Intelligence 90(1-2) (1997) 225-279

7. Dung, P.M.: On the acceptability of arguments and its fundamental role in nonmonotonic reasoning, logic programming and n-person games. Artif. Intell. 77(2) (1995) 321-357

8. Dung, P.M., Mancarella, P., Toni, F.: Computing ideal sceptical argumentation. Artificial Intelligence, Special Issue on Argumentation 171(10-15) (2007) 642-674

9. Vreeswijk, G., Prakken, H.: Credulous and sceptical argument games for preferred semantics. In: Proc. of the 7th European Workshop on Logic for Artificial Intelligence (JELIA). Number 1919 in Lecture Notes in AI, Berlin, Springer Verlag (2000) 239-253 
10. Gartner, D., Toni, F.: CaSAPI: a system for credulous and sceptical argumentation. In Simari, G., Torroni, P., eds.: Proc. of the Workshop on Argumentation for Nonmonotonic Reasoning (ArgNMR). (2007) 80-95

11. Dung, P.M., Kowalski, R.A., Toni, F.: Dialectic proof procedures for assumptionbased, admissible argumentation. Artificial Intelligence 170(2) (2006) 114-159

12. Fox, J., Parsons, S.: On using arguments for reasoning about actions and values. In Doyle, J., Thomason, R.H., eds.: Proc. of the Working Papers of the AAAI Spring Symposium on Qualitative Preferences in Deliberation and Practical Reasoning, Standford (1997) 55-63

13. Oliver, R.M., Smith, J.Q., eds.: Influence Diagrams, Belief Nets and Decision Analysis. John Wiley and Sons (1988)

14. Raz, J., ed.: Practical Reasoning. Oxford University Press, Oxford, UK (1978)

15. Amgoud, L., Kaci, S.: On the generation of bipolar goals in argumentation-based negotiation. In Rahwan, I., Moraitis, P., Reed, C., eds.: In Argumentation in MultiAgent Systems: State of the art survey. Number 3366 in LNAI. Springer-Verlag (2005) 192-207

16. Thomason, R.H.: Desires and defaults: A framework for planning with inferred goals. In: Proc. of the 7th International Confenrence on Principle of Knowledge Representation and Reasoning (KR). (2000) 702-713

17. Hulstijn, J., van der Torre, L.W.N.: Combining goal generation and planning in an argumentation framework. In: Proc. of the 9th International Workshop on Non-Monotonic Reasoning (NMR'04). (2004) 212-218

18. Rahwan, I., Amgoud, L.: An argumentation-based approach for practical reasoning. In: Proc. of the 5th International Joint Conference on Autonomous Agents and Multiagent Systems (AAMAS), Hakodate, Japan, ACM Press (2006) 347-354

19. Guillermo R. Simari, Alejandro J. Garca, M.C.: Actions, planning and defeasible reasoning. In: Proc. of the 10th International Workshop on Non-Monotonic Reasoning (NMR), Whistler BC, Canada (June 2004) 377-384

20. Kakas, A., Moraitis, P.: Argumentative-based decision-making for autonomous agents. In: Proc. of the 2nd International Joint Conference on Autonomous Agents and Multi-Agent Systems (AAMAS), ACM Press (2003) 883-890

21. Atkinson, K., Bench-Capon, T., McBurney, P.: Computational representation of practical argument. Synthese, special issue on Knowledge, Rationality and Action 152(2) (2006) 157-206

22. Ouerdane, W., Maudet, N., Tsoukias, A.: Arguing over actions that involve multiple criteria: A critical review. In Berlin, S., ed.: Proc. of the European Conference on Symbolic and Quantitative Approaches to Reasoning with Uncertainty (ECSQARU). Volume 4724 of Lecture Notes in Computer Science. (2007) 308-319

23. Amgoud, L., Prade, H.: Comparing decisions in an argumentation-based setting. In: Proc. of the 11th International Workshop on Non-Monotonic Reasoning (NMR), Session on Argumentation, Dialogue, and Decision Making, Lake District, UK (May-June 2006) 426-432

24. Amgoud, L., Prade, H.: Explaining qualitative decision under uncertainty by argumentation. In: Proc. of the 21st National Conference on Artificial Intelligence (AAAI), Boston (July 2006) 16-20 